This article was published as: Physica A: Statistical Mechanics and its Applications 498, 86-95, 2018 DOI: https://doi.org/10.1016/j.physa.2017.12.136 


\title{
On the theory of hysteretic magnetostriction of soft ferrogels.
}

\author{
Andrey Zubarev ${ }^{1}$, Dmitry Chirikov ${ }^{1}$, Gennady Stepanov ${ }^{2}$, Dmitry Borin ${ }^{3}$, M.T. Lopez-Lopez ${ }^{4,5}$ \\ ${ }^{1}$ Ural Federal University, 620000, Yekaterinburg, Russia \\ ${ }^{2}$ State Scientific Research Institute of Chemistry and Technology of Organoelement \\ Compounds 105118, Moscow, Russia \\ ${ }^{3}$ Technische Universität Dresden, Chair of Magnetofluiddynamics, Measuring and \\ Automation Technology, 01062, Dresden, Germany \\ ${ }^{4}$ Departamento de Física Aplicada, Facultad de Ciencias, Universidad de Granada, \\ Campus de Fuentenueva, 18071 Granada, Spain. \\ ${ }^{5}$ Instituto de Investigatacion Biosantaria ibs. GRANADA, Spain
}

\begin{abstract}
The paper deals with theoretical study of hysteretic magnetostriction of soft ferrogels - composite materials, consisting of the micron-sized magnetizable particles embedded into gel matrices. It is supposed that initially, before application of an external magnetic field, the particles are homogeneously and isotropically distributed in an elastic matrix. The theoretical explanation of the hysteresis phenomena is based on the conception that, under the field action, the particles rearrange into the linear chain-like aggregates. The typical length of the chains is determined by the competition between the force of magnetic attraction of the particles and the force of elastic deformation of the matrix.
\end{abstract}

Magnetic gels; magnetostriction; hysteresis

\section{Introduction}

The possibility to control physical properties and behavior of polymer and other soft matters by using magnetic fields is very attractive for many industrial and bio-medical applications. However, to the best of our knowledge, all natural soft matters are diamagnetic. Therefore, control of their properties requires very strong magnetic fields and bulky apparatuses. The effective solution of this problem gives the composites of fine (nano- and micron-sized) magnetic particles in polymer matrix. Coupling of high elasticity with high reaction on magnetic field gives new kind of smart composite materials, which find active applications in many industrial, medical and biological technologies. That is why they attract considerable interest of researches and engineers [1-13].

Experiments demonstrate hystereses of mechanic and magnetic phenomena in ferrogels with the micron-sized magnetizable particles (see, for example, [14-18]). The hysteretic effects present significant interest both from scientific point of view and viewpoint of practical applications of these composites. 
Analysis shows that rearrangement of the particles under the action of external magnetic field and their unification in heterogeneous structures can be one of internal causes of these hysteresis's. Note that appearance, under the field action, of the linear chain-like aggregates in soft polymerized gels has been directly observed in [15]. Another physical reason of the hysteretic phenomena can lie in disruption between particles and the polymer matrix [16]. However, in this case the hysteresis loops must be rather irreproducible - each new cycle of the field increase and decrease must correspond to new curves of the sample magnetization either deformation. At the same time, the results $[14,16]$ were quite repeatable for all circles of the applied field.

The hysteretic dependence of the gel magnetization on the external field has been explained in [19] on the basis of the concept of the particles unification into the linear chains while increase of the applied field and the chains rupture when the field is decreased. The aim of the present work is theoretical study of effect of the particles rearrangement on the hysteretic magnetostriction of the soft composites under the action of a uniform magnetic field. It should be noted, that experimentally detected magnetostriction in these composites, as a rule, is not large (relative elongation is about several per cent or a little more). That is why we restrict ourselves by analysis of only small deformations and suppose the linear relation between the sample deformation and corresponding elastic stress.

\section{Theoretical model and main approximations.}

We consider a ferrogel with micron-sized magnetizable particles and suppose that initially they are randomly (gas-like) distributed in a soft elastic matrix. Being magnetized by an external field, the particles unite into linear chains. We suppose also that the particles are strongly linked with the matrix, therefore, their motion through the matrix is impossible. That is why their unification into the chains is accompanied by the internal elastic deformation of the carrier polymer. As a consequence, the size of the chains is determined by the strength of the applied field, by the elastic properties of the matrix and specific of the initial spatial disposition of the particles.

Since the initial mutual disposition of the particles in the composite is random, the chains, under the given external conditions, must have various sizes with some function $g_{n}$ of distribution over the number $n$ of the particles in the chains. Strict determination of the function $g_{n}$ presents very complicated problem; its exact solution is unknown. To overcome the mathematical problem and to get the final results in a physically transparent form, we will use the hierarchical model suggested in [19], where the particles are distributed in a cubic cell (see Fig. 1) with the edge length:

$$
l=d\left(\frac{\pi}{6 \varphi}\right)^{\frac{1}{3}}
$$




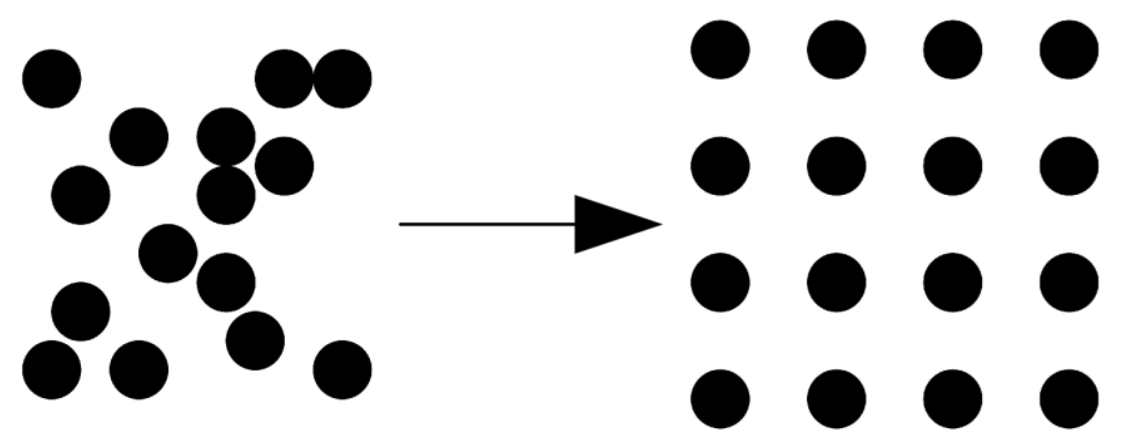

Figure 1. Illustration of the idea of the lattice model.

Here $d$ is the particle diameter, $\varphi$ is the particles volume concentration in the composite. The chosen edge length $l$ provides equality of the particles volume concentration in the model cubic cell to the real concentration $\varphi$. Magnetic field is supposed directed along one of the cell axes. Following to the hierarchical model [19], we will consider the process of the chain formation as the unification of the single particles into the doublets; then - unification of the doublets into the "quartets" of the particles, etc. Detailed description of the model can be found in [19]. We omit it here for brevity.

When the field is decreased, the chains disintegrate with the opposite way. This is of principally important that the disintegration of a chain takes place at lower field strength, than its formation [17]. This means that both, the distribution function $g_{n}$ and the mean number $\langle n\rangle$ of the particles in the chain hystereticaly depend on the applied magnetic field. In part, it leads to the hysteretic dependence of the composite magnetization as well as its susceptibility on the external field [19].

In spite of the strong simplifications, the model [19] reproduces the main physical features of experimental curves of the ferrogels magnetization, at least, in the frame of the correct order of magnitudes. That is why we will apply this approach to study the hysteretic magnetostriction of ferrogels.

\section{Free energy of the soft ferrogels.}

We will consider a cylindrical sample of the composite and suppose that a uniform magnetic field $H_{0}$ is applied along the cylinder axis. This situation is illustrated in Fig.2. Let us denote the length of the axis and diameter of the cylinder as $a$ and $b$ respectively. These magnitudes of the non deformed sample will be denoted as $a_{0}$ and $b_{0}$. The notation $r=a / b$ will be used for the sample aspect ratio. We will suppose that after
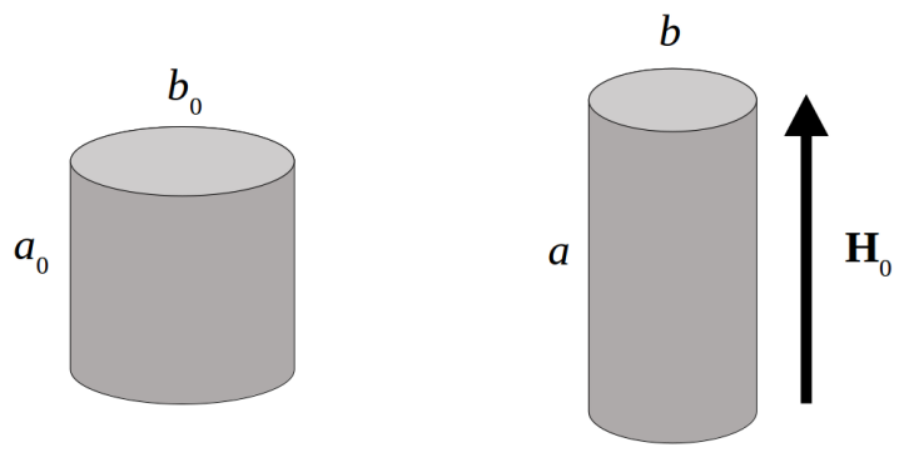

magnetostriction the sample also has the cylindrical shape with another value of the aspect ratio $r$.

Figure 2. Sketch of cylindrical sample. Left and right - before and after the magnetostriction respectively. 
For maximal simplification of the mathematical part of the problem, we will suppose that the soft polymer matrix is incompressible. It should be noted that this condition is fulfilled not for all gels [20]. Analysis of effect of the matrix compressibility can be considered as a generalization and continuation of this work.

Let $\varepsilon$ be a small relative elongation of the sample in the direction of the axis $a$. One can show easily that the following relations:

$$
a=a_{0}(1+\varepsilon), \quad b=\frac{b_{0}}{\sqrt{1+\varepsilon}} \approx b_{0}\left(1-\frac{\varepsilon}{2}\right) .
$$

are held for the incompressible sample. The positive sign of $\varepsilon$ corresponds to the sample elongation in the field direction; the negative one - to its contraction. The relative uniaxial deformation $\varepsilon$ of the sample can be estimated from the condition of minimum of free energy of the sample in the external magnetic field [21]. Estimation of the ferrogels free energy is the main goal of this part of the work.

Inhomogeneity of the magnetic field $H$ and magnetization $M$ inside the cylindrical sample makes the mathematical analysis of the problem very cumbersome and complicated. To overcome this problem, we will use the approximation [22] of the spatially uniform $H$ and $M$ equal to their average magnitude over volume of the cylinder. Of course, this is very strong simplification, however it allows us to use the results of numerical calculations [22] for the sample demagnetizing factor $N(r)$. It should be noted that for an ellipsoidal sample the demagnetizing shape-factor is determined only by the ellipsoid shape. For a cylinder the demagnetizing factor depends also on the sample magnetic susceptibility. The numerical results for $N(r)$ are tabulated in [22] for various magnitudes of $\chi$. These results very weakly depend on $\chi$ and their difference from $N(r)$, obtained for $\chi=0$, is practically negligible. That is why below, for simplicity, we will use the magnitudes of $N(r)$ calculated in [22] for $\chi=0$.

The density of the sample free energy $F$ can be presented as a sum of the densities of the magnetic $F_{m}$ and elastic $F_{e l}$ free energies [21,23]:

$$
F=F_{m}+F_{e l}
$$

The density of the magnetic free energy can be calculated as [21]:

$$
F_{m}=-\mu_{0} \int_{0}^{H_{0}} M\left(h_{0}\right) d h_{0} .
$$

Here $M\left(h_{0}\right)$ is magnetization of the sample placed in an external field $h_{0}$

Our first aim is to determine the sample magnetization $M$ as a function of the applied field. We will take into account that the particles in the chains are magnetized both by the magnetic field $H_{0}$ and due to effect of their mutual magnetization. Obviously, magnetic interaction between the closely situated particles in a chain is significantly stronger than this interaction between the particles which belong to the different chains. Thus, in the first approximation, one can determine magnetization of the sample $M$ taking into 
account magnetic interaction of the particles in one $n$-particle chain, neglecting interaction between particles in the different chains.

For the composite with the chain-like aggregates, one can use the following estimate [19]:

$$
M=\frac{\varphi \sum_{n=1}^{\infty} n M_{n} g_{n}}{\sum_{n=1}^{\infty} n g_{n}} .
$$

where $M_{n}$ is magnetization of a particle in the $n$-particle chain, $\varphi$ is volume concentration of the particles in the composite, $g_{n}$ is number of the $n$-particle chains per unit volume of the composite.

Let us introduce the dimensionless internal magnetic field and magnetization in the following way:

$$
\widetilde{H}=\frac{H}{M_{s}}, \quad \widetilde{M}=\frac{M}{M_{s}}, \quad \tilde{h}_{0}=\frac{h_{0}}{M_{s}}, \quad \widetilde{H}_{0}=\frac{H_{0}}{M_{s}} .
$$

Here $M_{s}$ is saturated magnetization of the particle material.

The particle magnetization $M_{n}$ was estimated in [19] by using the Frolich-Kennelly relation [24] between $M_{n}$ and magnetic field inside the particle in the approximation of the perfectly straight chains. The result reads:

$$
\begin{gathered}
\widetilde{M}_{n}=\frac{G_{n}-\sqrt{G_{n}^{2}-4 \chi_{p}^{2} \widetilde{H} f_{n}}}{2 \chi_{p} f_{n}}, \quad G_{n}=1+\chi_{p}\left(f_{n}+\widetilde{H}\right), \quad f_{n}=\frac{1}{3}\left(1-\frac{\Psi_{n}}{4 n}\right), \\
\Psi_{n}=2 \sum_{i=1}^{n-1} \frac{n-i}{i^{3}} .
\end{gathered}
$$

Here $\chi_{p}$ is initial magnetic susceptibility of the particle material.

Strictly speaking, magnetization of a particle in a chain depends on its position in the chain. The particle magnetization has been estimated in [19] in dependence on its position in the chain. The relation (5) presents the mean value of the particle magnetization, averaged over all particles in the $n$-particle chains. In [19] the force of magnetic interaction between the chains has been calculated in the approximations when the particle magnetization depends on its position in the chain as well as under assumption that magnetization of each particle equals to the mean magnetization (5). Results of both approximations are quite close. However, calculations in the approximation (5) of the mean magnetization are much less cumbersome. That is why here we use this approximation, i.e. eq. (5).

The distribution function $g_{n}$ also has been determined in [19] for the both cases, when applied magnetic field $H_{0}$ was increased and decreased. The relations [19] for $g_{n}$ are cumbersome and we omit them here.

Let us introduce the dimensionless density of the magnetic free energy:

$$
\tilde{F}_{m}=\frac{F_{m}}{E}=-\beta \int_{0}^{\widetilde{H}_{0}} \tilde{M} d \tilde{h}_{0}, \quad \beta=\frac{\mu_{0} M_{s}^{2}}{E} .
$$


Here $E$ is the Young's modulus of the pure matrix, $\beta$ is the parameter which defines the ratio of the energy of magnetic interaction between two magnetically saturated particles to the energy of elastic deformation of the matrix.

The following relation [21] takes place for a uniformly magnetizable sample in an external magnetic field $H_{0}$ :

$$
\widetilde{H}_{0}=\widetilde{H}+N \widetilde{M}
$$

therefore:

$$
d \widetilde{H}_{0}=d \widetilde{H}+N d \widetilde{M}
$$

Here $H$ is magnetic field inside the sample, $N$ is its demagnetizing factor.

Substituting (7) into the integral (6), we get:

$$
\tilde{F}_{m}=-\beta\left[\int_{0}^{\widetilde{H}} \tilde{M}(\tilde{h}) d \tilde{h}+\frac{N \widetilde{M}^{2}}{2}\right] .
$$

Here $M(\tilde{h})$ is magnetization of the sample when the internal field is $h$ and

$$
\widetilde{H}=\frac{H}{M_{s}}, \quad \tilde{h}=\frac{h}{M_{s}}
$$

Let us expand the density of the magnetic free energy in the power series with respect to the small relative elongation $\varepsilon$ of the sample. In the framework of the linear approximation:

$$
\tilde{F}_{m}(\varepsilon)=\tilde{F}_{m}(0)+\tilde{A} \varepsilon, \quad \tilde{A}=\left.\frac{\partial \tilde{F}_{m}}{\partial \varepsilon}\right|_{\varepsilon=0} .
$$

Here $\widetilde{F}_{m}(0)$ is the density of the free energy of the non deformed sample.

Now we will determine the coefficient $\tilde{A}$. Combining the second relation in (9) with eq. (8), after transformations one can obtain:

$$
\tilde{A}=\beta\left(\frac{N^{\prime} \widetilde{M}^{2}}{2}-\int_{0}^{\widetilde{H}} \widetilde{M}^{\prime} d \tilde{h}\right) .
$$

Here and below the prime means the differentiation over $\varepsilon$.

Simple calculations show that, in the linear approximation with respect to, the following relation

$$
N^{\prime}=\frac{d N}{d \varepsilon}=\frac{3 r}{2} \frac{d N}{d r}
$$

is held. The derivative $\widetilde{M}^{\prime}$ of the magnetization is presented below in eq. (26).

The derivative $d N / d r$ can be estimated by using numerical results for the cylinder shape-factor $N$, tabulated in [22].

Analysis shows that magnetic interaction between the chains must be taken into account for the adequate description of the hysteretic effects. We will consider interaction between the chains by using the regular pair approximation. This means, that interaction only between two chains will be taken into account; any effect of the third one will be neglected. The similar problem has been considered in [25] under 
assumption that all chains are identical. Here we will take into account that the chains can have different lengths.

Let $\mathrm{Wnm}(\mathbf{r})$ be the energy of the magnetic interaction between two chains, shown in Fig. 3; $\mathrm{q}(\mathbf{r})$ is the pair function of the spatial distribution of the chains, normalized so that $q \rightarrow 1$ when $r \rightarrow \infty$; the radiusvector $r$ links centers of the left particles of these chains.

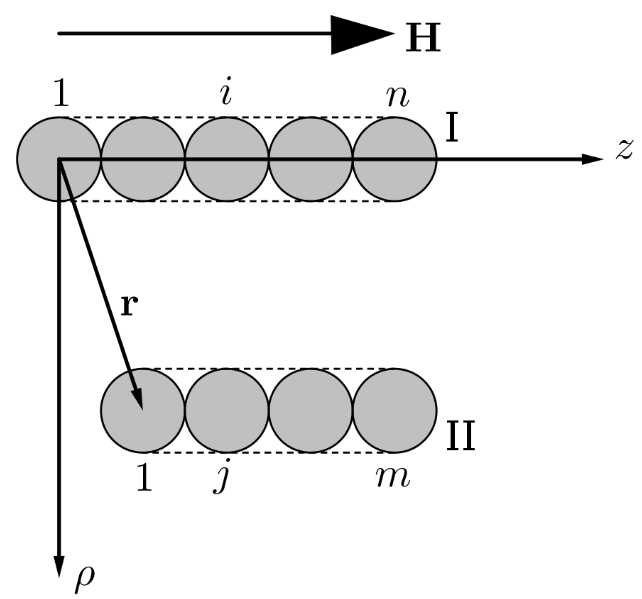

Figure 3. Sketch of the chains in the composition (cylindrical coordinates).

It should be noted that the real chains in magnetic gels are not perfectly straight. The shape of the chains is determined by details of initial disposition of the particles in the liquid polymer; by the magnitude of the field of polymerization and the rate of this process; by the interchain interaction and, possibly, by other factors. Here, like in ref. [19], we use the simplest approximation of the straight chains.

In the frames of the approximation of pair interchain interaction, the mean density energy $w$ of this interaction is:

$$
w=\frac{1}{2} \sum_{n=1}^{\infty} g_{n}^{2} \int W_{n n}(\mathbf{r}) q(\mathbf{r}) d \mathbf{r}+\sum_{n=2}^{\infty} \sum_{m=1}^{m<n} g_{n} g_{m} \int W_{n m}(\mathbf{r}) q(\mathbf{r}) d \mathbf{r} .
$$

We present the pair distribution function $q$ in the form:

$$
q=q_{0}+\delta q .
$$

Here $q_{0}$ is the function $q$ in the non deformed sample, $\delta q$ is the change of this function due to the sample deformation. In the pair approximation, the distribution function $q_{0}$ reads:

$$
q_{0}=\left\{\begin{array}{l}
0, \text { chains intersect } \\
1, \text { chains don't intersect }
\end{array}\right.
$$

In the linear approximation with respect to the small deformations $\varepsilon$, the term $\delta q$ can be determined from the following equation [26]:

$$
\delta q=-\operatorname{div}\left(q_{0} \mathbf{u}\right)=-q_{0} \operatorname{div} \mathbf{u}-(\mathbf{u} \cdot \nabla) q_{0} .
$$

Here $\mathbf{u}$ is the mean vector of the medium displacement. For the incompressible media the equality $\operatorname{div} \mathbf{u}=0$ is held, thus eq. (12) can be rewritten as:

$$
\delta q=-(\mathbf{u} \cdot \nabla) q_{0}
$$


Deformation of the sample leads to the change $\delta \mathrm{q}$ of the pair distribution function $\mathrm{q}$. In its turn that leads to the change of the density of the sample magnetic energy:

$$
\begin{gathered}
\delta w=\frac{1}{2} \sum_{n=1}^{\infty} g_{n}^{2} \int W_{n n}(\mathbf{r}) \delta q(\mathbf{r}) d \mathbf{r}+\sum_{n=2}^{\infty} \sum_{m=1}^{m<n} g_{n} g_{m} \int W_{n m}(\mathbf{r}) \delta q(\mathbf{r}) d \mathbf{r}= \\
=-\frac{1}{2} \sum_{n=1}^{\infty} g_{n}^{2} \int W_{n n}(\mathbf{r})(\mathbf{u} \cdot \nabla) q_{0} d \mathbf{r}-\sum_{n=2}^{\infty} \sum_{m=1}^{m<n} g_{n} g_{m} \int W_{n m}(\mathbf{r})(\mathbf{u} \cdot \nabla) q_{0} d \mathbf{r} .
\end{gathered}
$$

For convenience, we will introduce the following dimensionless quantities:

$$
\begin{gathered}
\widetilde{w}=\frac{\delta w}{E}, \quad \tilde{g}_{n}=\frac{g_{n} \varphi}{v_{p}}, \quad \widetilde{W}_{n m}=\frac{W_{n m}}{E d^{3}}, \quad \tilde{\mathbf{r}}=\frac{\mathbf{r}}{d}, \quad \tilde{\mathbf{u}}=\frac{\mathbf{u}}{d}, \quad v_{p}=\frac{\pi d^{3}}{6}, \\
\delta \widetilde{w}=-\frac{36 \varphi^{2}}{\pi^{2}}\left[\frac{1}{2} \sum_{n=1}^{\infty} \tilde{g}_{n}^{2} \int \widetilde{W}_{n n}(\widetilde{\mathbf{r}})(\tilde{\mathbf{u}} \cdot \nabla) q_{0} d \tilde{\mathbf{r}}+\sum_{n=2}^{\infty} \sum_{m=1}^{m<n} \tilde{g}_{n} \tilde{g}_{m} \int \widetilde{W}_{n m}(\tilde{\mathbf{r}})(\tilde{\mathbf{u}} \cdot \nabla) q_{0} d \tilde{\mathbf{r}}\right] .
\end{gathered}
$$

Here $\mathrm{d}$ and $v_{p}$ are diameter and volume of the particle respectively.

For maximal simplification of calculation of the integral (15), let us model the $n$-particle chain by the spherocylinder whose diameter and length of the cylindrical part equal to the particle diameter $d$ and $d(n-1)$ respectively. The spherocylinders, consisting of $n$ - and $m$-particles, are illustrated in Fig. 3 by the dashed lines. In this case the gradient $\nabla q_{0}$ is proportional to $\delta\left(\mathbf{r}-\mathbf{r}_{s}\right)$, where $\delta(x)$ is the delta-function, and $\mathbf{r}_{s}$ is the radius-vector, corresponding to the boundary of the region of the spherocylinders intersection. One can show easily that, the surface, corresponding to $\mathbf{r}_{s}$, presents a spherocylinder whose diameter and length of the cylindrical part equal to the particle diameter $2 d$ and $d(n+m-2)$ respectively.

Neglecting the effect of the interchain interaction on magnetic moments of the particles in the chains, one can present the energy $\widetilde{W}_{n m}$ of interaction between two chains as:

$$
\widetilde{W}_{n m}=\frac{\pi \beta \widetilde{M}_{n} \widetilde{M}_{m}}{144} \sum_{i=1}^{n} \sum_{i=1}^{m} \frac{\tilde{\rho}^{2}-2(j-i+\tilde{z})^{2}}{\left[\tilde{\rho}^{2}+(j-i+\tilde{z})^{2}\right]^{\frac{5}{2}}}, \quad \tilde{z}=\frac{z}{d}, \quad \tilde{\rho}=\frac{\rho}{d}
$$

Here $i$ and $j$ are the numbers of particles in chains I and II in Fig. 3; $z$ and $\rho$ are cylindrical coordinates of the center of the left particle (particle with the number 1) of the chain II.

Let us present the dimensionless change (15) of the magnetic energy density $w$, defined in (11), as:

$$
\delta \widetilde{W}=-\beta \varepsilon \varphi^{2}\left(\frac{1}{2} \sum_{n=1}^{\infty} \tilde{g}_{n}^{2} \widetilde{M}_{n}^{2} J_{n n}+\sum_{n=2}^{\infty} \sum_{m=1}^{m<n} \widetilde{g}_{n} \widetilde{g}_{m} \widetilde{M}_{n} \widetilde{M}_{m} J_{n m}\right), \quad J_{n m}=J_{n m}^{(1)}+J_{n m}^{(2)} .
$$

Here $J_{n m}^{(1)}$ corresponds to integration in eq. (15) over the cylindrical part of the spherocylinder excluded for the second chain (the surface of the excluded spherocylinder with diameter $2 d$ and length of the cylindrical part equal to $d(n+m-2)) ; J_{n m}^{(2)}$ - to the integration over the semispheres of this spherocylinder. Parameter $\beta$ is defined in eq. (6).

For the small uniaxial stretching, corresponding to eq. (1), in a Cartesian coordinate system with the origin in the center of the left particle of the chain I in Fig. 3, the components of the vector $\mathbf{u}$ are: 


$$
u_{z}=\varepsilon z, \quad u_{x}=-\frac{\varepsilon x}{2}, \quad u_{y}=-\frac{\varepsilon y}{2} .
$$

The axis $O z$, like in Fig. 3, is aligned along the internal field H. In the cylindrical coordinate system, shown in Fig.3, the non-zero components of this vector are:

$$
u_{z}=\varepsilon Z, \quad u_{\rho}=-\frac{\varepsilon \rho}{2} .
$$

Combining Eqs. (15) and (17), we can write down the integral $J_{\mathrm{nm}}^{(1)}$ in the following form:

$$
J_{n m}^{(1)}=-\frac{1}{4} \sum_{i=1}^{n} \sum_{j=1}^{m} \int_{1-m}^{n-1} \frac{\tilde{\rho}^{2}-2(j-i+\tilde{z})^{2}}{\left[\tilde{\rho}_{2}+(j-i+\tilde{z})^{2}\right]^{\frac{5}{2}}} d \tilde{z} .
$$

Here we take into account that the integral (19) must be calculated over the cylindrical part of the surface $\mathbf{r}_{s}$, i.e. at $\rho=d$. This integral can be calculated analytically:

$$
\begin{gathered}
J_{n m}^{(1)}=\frac{1}{4} \sum_{i=1}^{n} \sum_{j=1}^{m}\left\{\frac{1-m+j-i}{\left[1+(1-m+j-i)^{2}\right]^{\frac{3}{2}}}-\frac{n-1+j-i}{\left[1+(n-1+j-i)^{2}\right]^{\frac{3}{2}}}\right\}, \\
J_{n n}^{(1)}=-\frac{1}{2}\left\{\frac{n(n-1)}{\left(n^{2}-2 n+2\right)^{\frac{3}{2}}}+\sum_{i=1}^{n-1}(n-i)\left[\frac{n-1-i}{\left[1+(n-1-i)^{2}\right]^{\frac{3}{2}}}+\frac{i+n-1}{\left[1+(i+n-1)^{2}\right]^{\frac{3}{2}}}\right]\right\} .
\end{gathered}
$$

In order to calculate $J_{n m}^{(2)}$, for convenience, we will use the spherical coordinate system, shown in Fig. 4 with the radius $r$ and polar angle $\theta$.

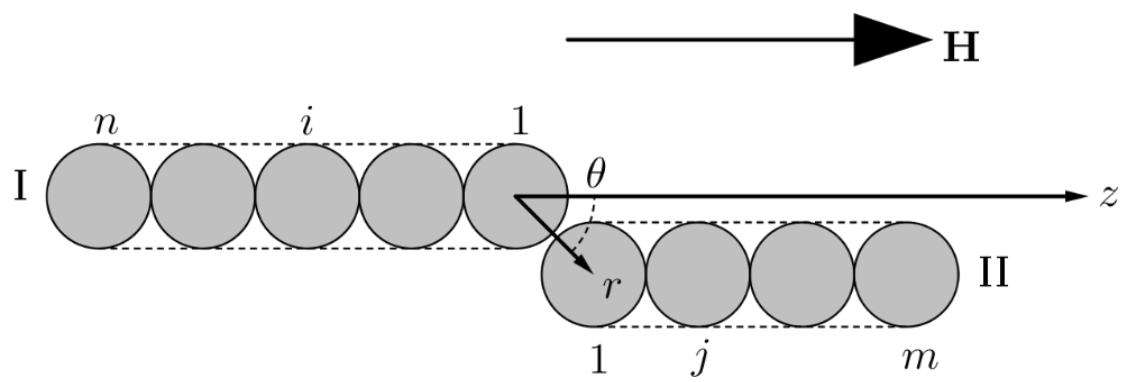

Figure 4. Illustration of the spherical coordinate system.

In this system the radial component of the vector $\mathbf{u}$ is:

$$
u_{r}=\frac{\varepsilon r\left(3 \cos ^{2} \theta-1\right)}{2}
$$

The integral $J_{n m}^{(2)}$ reads:

$$
J_{n m}^{(2)}=\frac{1}{2} \sum_{i=1}^{n} \sum_{j=1}^{m} \int_{0}^{\frac{\pi}{2}} \frac{\sin ^{2} \theta-2(i+j-2+\cos \theta)^{2}}{\left[(i+j-2+\cos \theta)^{2}+\sin ^{2} \theta\right]^{\frac{5}{2}}}\left(3 \cos ^{2} \theta-1\right) \sin \theta d \theta
$$

Taking into account that the spherocylinder has two semispheres, after simple, but cumbersome calculations, one can get the following analytical expression for the integral (22): 


$$
\begin{gathered}
J_{n m}^{(2)}=\frac{1}{10} \sum_{j=1}^{m-1} \frac{1}{j^{5}}\left[\frac{4 j^{8}+6 j^{6}+9 j^{4}+36 j^{2}+24}{\left(j^{2}+1\right)^{\frac{3}{2}}}-24\right]+ \\
+\frac{1}{10} \sum_{i=1}^{n-1} \sum_{j=0}^{m-1} \frac{1}{(i+j)^{5}}\left[\frac{4(i+j)^{8}+6(i+j)^{6}+9(i+j)^{4}+36(i+j)^{2}+24}{\left[(i+j)^{2}+1\right]^{\frac{3}{2}}}-24\right]-\frac{2 n m}{5} \\
J_{n n}^{(2)}=\frac{1}{10} \sum_{i=1}^{n-1} \frac{i+1}{i^{5}}\left[\frac{4 i^{8}+6 i^{6}+9 i^{4}+36 i^{2}+24}{\left.\left(i^{2}+1\right)^{\frac{3}{2}}-24\right]+}\right. \\
+\frac{1}{10} \sum_{i=n}^{2(n-1)} \frac{2 n-i-1}{i^{5}}\left[\frac{4 i^{8}+6 i^{6}+9 i^{4}+36 i^{2}+24}{\left(i^{2}+1\right)^{\frac{3}{2}}}-24\right]-\frac{2 n^{2}}{5} .
\end{gathered}
$$

In order to determine the coefficient $\tilde{A}$ in (10), we have to calculate the integral $\int_{0}^{\widetilde{H}} \widetilde{M}^{\prime} d \widetilde{H}$. It can be expressed via change of the dimensionless magnetic free energy as follows:

$$
\delta \widetilde{W}=-\beta \int_{0}^{\widetilde{H}} \delta \widetilde{M} d \tilde{h}=-\beta \varepsilon \int_{0}^{\widetilde{H}} \widetilde{M}^{\prime} d \tilde{h}
$$

One needs to note that $\delta w$ is the change of the sample magnetic free energy only due to the change of the chains mutual disposition as a consequence of the sample deformation. The effect of variation of the sample demagnetizing factor $N$ is not taken into account here.

Now we are in position to get the explicit form for the derivative of magnetization $\widetilde{M}$ over elongation $\varepsilon$. Combining the first relation in (17) with (24), one gets:

$$
\int_{0}^{\widetilde{H}} \widetilde{M}^{\prime} d \tilde{h}=-\frac{\delta \widetilde{w}}{\beta \varepsilon}=\varphi^{2}\left(\frac{1}{2} \sum_{n=1}^{\infty} \tilde{g}_{n}^{2} \widetilde{M}_{n}^{2} J_{n n}+\sum_{n=2}^{\infty} \sum_{m=1}^{m<n} \tilde{g}_{n} \widetilde{g}_{m} \widetilde{M}_{n} \widetilde{M}_{m} J_{n m}\right) .
$$

Differentiating left and right parts of (25) over magnetic field inside of the sample $\widetilde{H}$, we obtain the following relation for $\tilde{M}^{\prime}$ :

$$
\begin{gathered}
\widetilde{M}^{\prime}=\varphi^{2}\left[\sum_{n=1}^{\infty} \tilde{g}_{n}^{2} \widetilde{M}_{n} \frac{\partial \widetilde{M}_{n}}{\partial \widetilde{H}} J_{n n}+\sum_{n=2}^{\infty} \sum_{m=1}^{m<n} \tilde{g}_{n} \tilde{g}_{m}\left(\widetilde{M}_{n} \frac{\partial \widetilde{M}_{m}}{\partial \widetilde{H}}+\widetilde{M}_{m} \frac{\partial \widetilde{M}_{n}}{\partial \widetilde{H}}\right) J_{n m}\right], \\
\frac{\partial \widetilde{M}_{n}}{\partial \widetilde{H}}=\frac{1}{2 f_{n}}\left(1-\frac{1+\chi_{p} \widetilde{H}-\chi_{p} f_{n}}{\sqrt{G_{n}^{2}-4 \chi_{p}^{2} \widetilde{H} f_{n}}}\right) .
\end{gathered}
$$

Combining (10), (15) and (24), we get:

$$
\tilde{A}=\beta\left[\frac{3 r \widetilde{M}^{2}}{4} \frac{d N}{d r}-\varphi^{2}\left(\frac{1}{2} \sum_{n=1}^{\infty} \tilde{g}_{n}^{2} \widetilde{M}_{n}^{2} J_{n n}+\sum_{n=2}^{\infty} \sum_{m=1}^{m<n} \tilde{g}_{n} \tilde{g}_{m} \widetilde{M}_{n} \widetilde{M}_{m} J_{n m}\right)\right] .
$$

Substituting (27) into (9), we estimate the change of the magnetic free energy due to the sample deformation.

In the case of the small deformation $\varepsilon$, the dimensionless elastic free energy can be presented as [26]: 


$$
\tilde{F}_{e l}=\frac{\tilde{E}_{c} \varepsilon^{2}}{2}
$$

Here $\widetilde{E}_{c}$ is the dimensionless Young's modulus of the composite with the spherical hard particles.

For the dilute composites with the spherical particles, where their elastic interaction due to perturbations of the matrix can be ignored, the modules can be calculated mathematically strictly. For the concentrated systems, where these interactions are significant, the mathematically strict methods have not been developed yet. That is why various empirical and semi-empirical relations are used for the mechanical modulus of the composite materials (see discussion in [28]).

It was shown in [28] that the empirical expression [29]:

$$
\tilde{E}_{c}=\frac{2+\varphi}{2(1-2 \varphi)}
$$

quite well describes experiments with elastic composites. It should be noted that appearance of the chains can lead to significant increase of the elastic modulus of magnetic gels [30,31]. Unfortunately, to the best of our knowledge, analytical expressions, describing this effect, have not been obtained in literature. That is why we will use here estimate (29) for the Young's modulus of the composite.

\section{Results and discussion.}

Substituting Eqs. (9), (28) into (2), we come to the following relation for the total density of the sample free energy at the small deformation $\varepsilon$ :

$$
\tilde{F}=\tilde{F}_{m}(0)+\tilde{A} \varepsilon+\frac{\tilde{E}_{c} \varepsilon^{2}}{2}
$$

The equilibrium deformation $\varepsilon$ of the incompressible sample corresponds to the minimum of its free energy. By using (30), we get:

$$
\varepsilon=-\frac{\tilde{A}}{\widetilde{E}_{c}} .
$$

Our results of calculations of the sample deformation and comparison of the theoretical data with experiments [16] are shown in Fig. 5. 

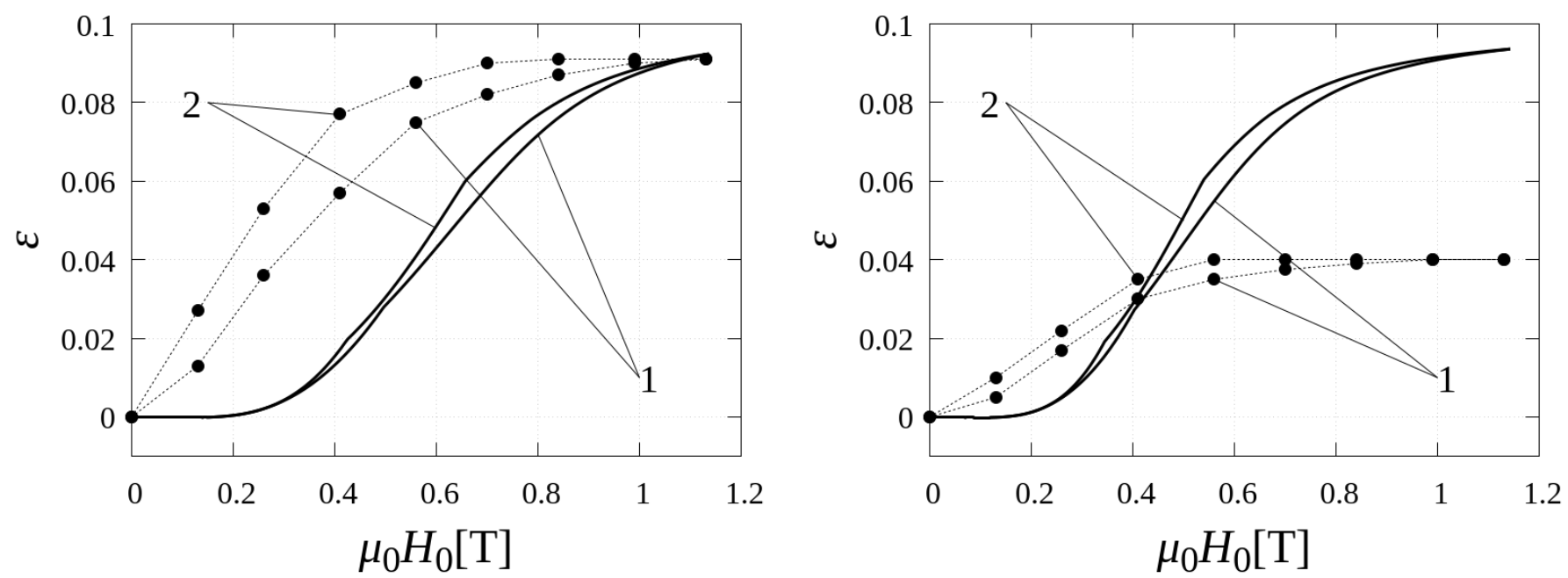

Figure 5. Relative strain $\varepsilon$ of a ferrogel vs. applied magnetic field $H_{0}$. Lines - theory, dots - experiment [16]. Line 1 - magnetic field is increased; line $2-$ magnetic field is decreased. System parameters: volume concentration of the particles $\varphi=0.30$; elastic modulus of the matrix $E=140 \mathrm{kPa}$; initial susceptibility of the particles material $\chi_{p}=100$; saturated magnetization of the particle material $M_{s}=1650 \mathrm{kA} / \mathrm{m}$; the aspect ratio of the non deformed sample $r=0.33$ (left figure) and $r=1.03$ (right figure).

In the order of magnitude the theoretical results are in agreement with the experimental ones. However, the experimentally detected deformation $\varepsilon$ much stronger depends on the sample aspect ratio $r$ than the theory predicts. It should be noted that the sample shape (the aspect ratio $r$ ) effects on the magnetostriction, first of all, through the magnetic field $\mathrm{H}$ inside the sample; this field is determined by the demagnetizing shape-factor $N$. The sample deformation changes its free energy density $F$; the corresponding term A in $(9,10,27)$ contains the multiplier $r \frac{d N}{d r}$. At the large field $\mathrm{H}_{0}$, when the magnetization $\mathrm{M}$ is saturated, only the term $r \frac{d N}{d r}$ determines dependence of the deformation on the aspect ratio. In the range of $r$, corresponding to Fig. $5(0.33<r<1.03)$, the multiplication $r \frac{d N}{d r}$ weakly depends on $r$. That is why the theoretical results in the left and right parts of Fig.5 are very close. The theoretical dependence of the deformation $\varepsilon$ on the aspect ratio $r$ through the multiplication $r \frac{d N}{d r}$ follows from the general thermodynamic considerations, similar to those in [21] at the calculation of striction of a linearly polarized ellipsoid under the action of external field.

We think that the problem of this disagreement between the results of the thermodynamic analysis and measurements of [16] requires the further experimental and theoretical study.

For small and high magnetic fields the theoretical hysteretic loops are very narrow and the difference between the curves, corresponding to the increase and decrease of the field, is practically invisible in Fig. 5. The reason of the small alternation, of course, is in the used simplifications (the initial cubic disposition of the particles; the hierarchic model of the linear chains formation; their perfectly straight shape). Unfortunately, refusal to these simplifications leads to very cumbersome calculations and non transparent form of the final results. That is why here we restricted ourselves by consideration and analysis only those physical factors which are principally important for theoretical description of the hysteretic phenomena.

Note also that the volume concentration of the particles in the samples, used in the experiments [16], was about thirty per cent. In the systems with the concentrations as high as that, the separate linear chains 
hardly can appear - one can expect unification of the particles into topologically complicated anisotropic structures. Nevertheless, the present model, in spite of its simplicity, reproduces the main physical features of the experimental results - the hysteretic behavior of the elongation $\varepsilon$ as a function of the applied field $H_{0}$, as well as the tendency of the elongation to saturation while the field increasing. In the order of magnitude our results are in agreement with the experimental ones; no fit parameters have been used in our calculations. Therefore, the present model can be considered as a robust basement for modeling of the magnetomechanic effects in the soft magnetic gels.

\section{Conclusion}

We present a theoretical model of the hysteretic dependence of a ferrogel magnetostriction on the applied magnetic field. This hysteresis is explained by the unification of the particles into linear chain-like aggregates when the field is increased and by the rupture of the chains when the field is decreased. The obtained theoretical results, without any fitting parameters, reproduce the hysteretic effect; in the order of magnitude they are in agreement with experiments [16]. The quantitative discrepancy between the theoretical and experimental results can be explained, first of all, by the fact that the model deals with the linear chains, as the simplest kind of anisotropic heterogeneous structures, which can appear in the systems of magnetizable particles under the field action. The separate chains, in the systems of magnetizable particles, are expected when their concentration is low or moderate, when the lateral aggregation of the chains can be neglected (see, for example, [32,33]). In contrast, the experiments [16] have been performed with the highly concentrated composites, where appearance of topologically complicated structures is quite probable. Nevertheless, the model describes experiments [16], at least, in the order of magnitude. This allows us to consider the model as a robust background for the further study of more concentrated systems.

Unfortunately, we don't know the experimental results, obtained with the lower, that in [16], concentrated composites, to compare them with our model.

One needs to note that other mechanisms, besides considered here, can affect mechanical properties and behavior of the composite material, such as unbound the matrix macromolecules from the particles surface; reorganization of the polymer around the particles, etc. These factors require detailed experimental and theoretical studies and can be subjects of separate works.

\section{Acknowledgements}

This work has been done under the financial support of the Russian Fund for Basic Research, 16-5812003, 16-53-12009, 16-32-00019 mol a, the Program of Russian Federation Ministry of Science and Education, projects 3.1438.2017/4.6, 11.10010.2017/5.2; D.B. would like to acknowledge the financial support of the Deutsche Forschungsgemeinschaft (DFG) under Grant Bo 3343/1-1 within PAK 907. M.L-L was supported by the project FIS2013-41821-R (Plan Nacional de Investigación Científica, Desarrollo e 
Innovación Tecnológica, Ministerio de Economía y Competitividad, Spain, co-funded by ERDF, European Union).

\section{References}

[1] Filipcsei G, Csetneki I, Szilagyi A and Zrinyi M, Advanced Polymer Science, 206 (2007) 137-189.

[2] Boczkowska A and Awietjan S, Materials Science Forum, 636-637 (2010) 766-771.

[3] Dyke S, Spencer B and Sain M, Carlson J, Smart Materials and Structures, 5 (1996) 565-575.

[4] Occhiuzzi A, Spizzuoco M and Serino G, Smart Materials and Structures, 12 (2003) 703-711.

[5] Carmona F and Mouney C, Journal. Materials Science, 27 (1992) 1322-1326.

[6] Feller J, Linossier I and Grohens Y, Material Letters, 57 (2002) 64-71.

[7] Lundberg B and Sundqvist B Journal Applied Physics, 60 (1986) 1074 -1079.

[8] Jin S, Mottine J, John J and Sherwood R, U.S. Patent. 4 (1987) 644, 101.

[9] Barkauskas J, Talanta, 44 (1997) 1107-1112.

[10] Kim Y, Ha S, Yang Y, Kim Y, Cho S, Yang S and Kim Y, Sensors and. Actuators B, 108 (2005) 285-291.

[11] Bhattacharya S Metal Filled Polymers, New York: Marcel-Dekker, 1992.

[12] Fulton J, Moore R, Lambert W and Mottine J. Proc. 39 ${ }^{\text {th }}$ Electronic Components, Conf. IEEE 39, 71. 1989.

[13] Wood D. S. and Camp P. J., Phys. Rev. E, 83, (2011 )011402.

[14] Stepanov G, Abramchuk S, Grishin D, Nikitin L, Kramarenko E, Khokhlov A Polymer, 48 (2007) 488495.

[15] Stepanov G, Borin D, Raikher Yu, Melenev P, Perov N, J. Physics: Condensed Matter, 20 (2008) 204121.

[16] Diguet G, Beaugnon E and Cavaille J, Journal Magnetism and Magnetic Materials, 322 (2010) 3337 3341.

[17] Gundermann T, Cremer P, Löwen H, Menzel A M, Odenbach S, Smart Mater. Struct. 26 (2017) 045012.

[18] Schümann M, Odenbach S, Journal of Magnetism and Magnetic Materials 441 (2017) 88-92.

[19] Zubarev A, Chirikov, Borin D and Stepanov G, Soft Matter 12 (2016) 6473-6480.

[20] Shankar A., Safronov A., Mikhnevich E., Beketov I., Kurlyandskaya G., SoftMatter, 13 (2017) 3359

[21] Landau L, Lifshitz E, Electrodynamics of Continuum Media, Pergamon Press, London 1960.

[22] Chen D, Brug J and Goldfarb R. IEEE Transactions on Magnetics, 27 (1991) 3601-3619.

[23] Zubarev A, Physica A, 413, (2014) 400-408.

[24] Bozorth R, Ferromagnetism, Wiley, New York, 1993.

[25] Zubarev A, Physica A, 392, (2013) 0 4824-4836.

[26] Shkel Y, Klingenberg D, Journal Applied Physics 83, (1998) 7834-7843.

[27] Landau L, Lifshitz E, Theory of Elasticity, Pergamon Press, London 1970.

[28] Christensen R, Mechanics of Composite Materials, Dover Publication 2005. 
[29] Krieger I, Advanced Colloid and Interface Science, 3, (1972) 111-136.

[30] Pessot G., Löwen H., Menzel A.M, The Journal of Chemical Physics 145, (2016 )104904.

[31] Schümann M., Borin D. Y., Huang S., Auernhammer G. K., Müller R., Odenbach S., Smart Mater. Struct. 26 (2017) 095018

[32] Bossis G., Volkova O., Lacis S., and Meuner A., in Ferrofluids, Magnetically Controllable Fluids and Their Application (Ed. Odenbach S). Springer, Berlin, 2002.

[33] Bossis G., Lançon P., Meunier A. Iskakova L., Kostenko V. and Zubarev A., Physica A., 392 (2013) $1567-1576$. 